EMPIRISChe PolizeIforschung 


\title{
EMPIRISCHe Polizeiforschung
}

\author{
Interdisziplinäre Perspektiven \\ in einem sich entwickelnden Forschungsfeld
}

\author{
Karlhans Liebl \\ Thomas Ohlemacher (Hg.)
}

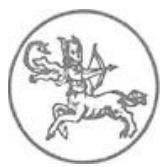

Centaurus Verlag \& Media UG 2000 
Die Herausgeber:

Prof. Dr. Karlhans Liebl ist Professor für Kriminologie an der Fachhochschule für Polizei Sachsen (Rothenburg).

PD Dr. Thomas Ohlemacher ist wissenschaftlicher Mitarbeiter am Kriminologischen Forschungsinstitut Niedersachsen (Hannover) und Privatdozent am Institut für Soziologie der Universität Hamburg.

Die Deutsche Bibliothek - CIP-Einheitsaufnahme

Empirische Polizeiforschung:

interdisziplinäre Perspektiven

in einem sich entwickelnden Forschungsfeld/

Karlhans Liebl / Thomas Ohlemacher ( $\mathrm{Hg}$.). -

Herbolzheim : Centaurus-Verl.-Ges., 2000

ISBN $978-3-8255-0314-7$

ISBN $978 \cdot 3-86226-877-1$ (eBook)

DOI $10.1007 / 978-3-86226-877-1$

Alle Rechte, insbesondere das Recht der Vervielfältigung und Verbreitung sowie der Übersetzung, vorbehalten. Kein Teil des Werkes darf in irgendeiner Form (durch Fotokopie, Mikrofilm oder ein anderes Verfahren) ohne schriftliche Genehmigung des Verlages reproduziert oder unter Verwendung elektronischer Systeme verarbeitet, vervielfältigt oder verbreitet werden.

C CENTAURUS Verlags-GmbH \& Co. KG, Herbolzheim 2000

Umschlaggestaltung: DTP-Studio, Antje Walter, Lenzkirch Umschlagabbildung: Eine Fußstreife vor dem „Bonner Loch“, einer stark frequentierten Geschäfts-, Bundes- und U-Bahn Unterführung. Aufnahme: Susanne Baumgarten. ( ) photoagentur vario-press. Das Photo wurde zu Verfügung gestellt vom Süddeutschen Verlag-Bilderdienst. 


\section{Inhaltsverzeichnis}

Empirische Polizeiforschung: Forschung in, für und über die Polizei (Thomas Ohlemacher und Karlhans Liebl).

\section{Polizei und Polizeikultur}

Cop Culture und Polizeikultur: Von den Schwierigkeiten einer Corporate Identity der Polizei (Rafael Behr)

Anforderungsprofile für Polizeibeamtinnen und Polizeibeamte, oder:

Was erwarten Polizistinnen und Polizisten von der Soziologie?

(Hans Schneider)

Mitarbeiterzufriedenheit in der Polizei: Weg von der abstrakten Beschreibung, hin zur konkreten Veränderung (Manfred Bornewasser)

\section{Konstruktionen von Kriminalität}

Erfahrungen, Einstellungen und Entscheidungsfindung in Betäubungsmittelstrafsachen bei der Polizei - Ausgewählte Befunde einer kriminologischen Untersuchung polizeilicher Rechtsanwendung (Jürgen Stock). .50

Fehlerquellen der Polizeilichen Kriminalstatistik (Willi Stadler und Werner Walser).

\section{Polizei und Bevölkerung}

Entwicklung des Vertrauens der Bevölkerung in die Polizei 1984-1995

(Kurt H. G. Groll und Bettina Lander)..

Polizeipräsenz und Sicherheitsgefühl: Eine vergleichende Analyse auf Basis von Aggregat- und Individualdaten (Karl-Heinz Reuband)...

Wer kontrolliert die Qualität der polizeilichen Arbeit? Subjektive Kontrollwahrnehmungen und Fremdenabweisung Hamburger Polizisten und Polizistinnen (Rainer Dollase).

Kriminologische Regionalanalyse Castrop-Rauxel: Quantitative und qualitative Ergebnisse im Überblick (Gisbert van Elsbergen) 
Polizeialltag und Habitus: Eine sozialökologische Fallstudie (Jörg Hüttermann).

Innovative Vorhaben

Evaluierung eines Fortbildungsprogramms für Trainer in Konflikthandhabung und Krisenintervention bei der Polizei Baden-Württemberg

(Werner K. Kunisch)

Das Projekt Jugendkriminalität in Kassel (Kai Freitag).

Kommunale Kriminalprävention in Deutschland. Eine quantitative Annäherung (Martina Kant, Norbert Pütter und Christine Hohmeyer).

Polizei im Wandel: Eine geplante empirische Analyse zur Arbeitssituation von Polizeibeamten und -beamtinnen in Niedersachsen

(Thomas Ohlemacher, Christiane Bosold und Christian Pfeiffer).

Verzeichnis der Autorinnen und Autoren 